\title{
Incidence, Management, and Cost of Complications After Transforaminal Endoscopic Decompression Surgery for Lumbar Foraminal and Lateral Recess Stenosis: A Value Proposition for Outpatient Ambulatory Surgery
}

\author{
KAI-UWE LEWANDROWSKI, MD \\ Center for Advanced Spine Care of Southern Arizona, Tucson, Arizona
}

\begin{abstract}
Objective: The objective of this study is to analyze incidence, estimate cost savings, and evaluate best management practices of complications resulting from outpatient transforaminal endoscopic decompression surgery for lumbar foraminal and lateral recess stenosis performed in an ambulatory surgery center.

Background: Endoscopic spinal surgery is gaining popularity for the treatment of lumbar disc herniations. Recent advances in surgical techniques allow for endoscopically assisted bony decompression for neurogenic claudication symptoms due to spinal stenosis. Postoperative complications from dural tears, recurrent disc herniations, nerve root injuries, foot drop, and facet and pedicle fractures, and postoperative sequelae such as dysesthetic leg pain and infiltration of the surgical access and spinal canal with irrigation fluid causing spinal headaches and painful wound swelling, as well as failure to cure, have been reported.

Methods: A retrospective study of 1839 consecutive patients with an average mean follow up of 33 months (range: 24 to 85 months) that underwent transforaminal endoscopic decompression surgery at 2076 levels between 2006 and 2015 was conducted to analyze incidence, and estimate the cost savings of postoperative adverse events following endoscopic foraminotomy and microdiscectomy. Complications were stratified using Dindo's 7-category grading system, distinguishing them from procedure-inherent sequelae as well as failure to cure. Only patients with unilateral radiculopathy due to either herniated disc or lateral recess stenosis were included in this study. Preoperatively, disc migration was graded by direction and distance from the disc space according to Lee's radiologic 4-zone classification. The type of disc herniation was classified either as contained or extruded. Contained herniations were further subclassified as disc protrusions versus disc bulges. In addition, the preoperative disc height was recorded. Bony spinal foraminal stenosis and lateral recess stenosis were graded on preoperative magnetic resonance imaging and computed tomography scans into mild, moderate, and severe by dividing the lumbar neuroforamen into 3 zones: (1) entry zone, (2) midzone, and (3) exit zone. Surgical outcomes were classified according to the Macnab criteria. In addition, reduction in the visual analog scale (VAS) scores were assessed.

Results: According to the Macnab criteria, excellent and good results were obtained in $82.2 \%$ of patients with extruded disc fragment (331/1839). In this group, the mean VAS score decreased from $5.9 \pm 2.5$ preoperatively to $2.4 \pm 1.8$ at final follow-up $(P<.01)$. Patients with contained disc herniations $(648 / 1839)$ had excellent and good results $72.7 \%$ of the time. In this group, the mean VAS score decreased from $7.2 \pm 1.6$ preoperatively to $3.1 \pm 1.5$ at final follow-up $(P<.01)$. In the spinal stenosis group $(860 / 1839), 75 \%$ of patients had excellent to good results. Postoperative grade I complications (any deviation from normal postoperative course treated with observation) occurred in 2 patients who immediately developed foot drop postoperatively on the surgical side $(0.11 \%)$ and in another 2 patients $(0.11 \%)$ with incidental durotomy. Grade II complications (any deviation with pharmacological interventions) occurred in 11 patients due to chronic obstructive pulmonary disease exacerbation, and in another 2 patients due to infections as the latter were successfully treated with antibiotics. Grade IIIb complications (any deviation requiring surgical, endoscopic, or radiological intervention under general anesthesia) occurred in 9 patients with reherniations of extruded discs within the first 3 postoperative months (recurrence rate $2.7 \%$ ). Reherniations were associated with preserved disc height of $>6 \mathrm{~mm}(P<.02)$. Grade IV (organ failure), and grade V (death) complications did not occur. Procedure-inherent sequelae from adverse operative side effects were noted in 8 patients with spinal headaches $(0.44 \%)$, and in 69 patients $(3.75 \%)$, who had extravasations of irrigation fluid into the subcutaneous tissues causing wound swelling. Another 229 patients developed postoperative dysesthetic leg pain due to irritation of the dorsal root ganglion $(12.45 \%)$, which was associated with severe foraminal stenosis $(P<.01)$ and improved with supportive care in all cases. Failure to cure occurred in 39 patients $(2.12 \%)$ with bony stenosis in the central canal, and lateral recess involving the entry zone of the neuroforamen and in 41 patients $(2.23 \%)$ with contained disc herniations.
\end{abstract}


Conclusions: Complications after outpatient transforaminal endoscopic decompression surgery with respect to reherniation, wound infections, durotomy, and nerve root injury are approximately 1 magnitude lower than equivalent reported complication rates with microdiscectomy while delivering comparable clinical outcomes and lower readmission rates to an emergency room or hospital. Postoperative sequelae are typically self-limiting and successfully managed with supportive care measures. Significant cost savings are realized due to a considerably lower rate of decompensated postoperative medical problems.

Endoscopic Minimally Invasive Surgery

Keywords: lumbar endoscopic, transforaminal decompression, complications, cost

\section{INTRODUCTION}

Microdiscectomy is considered the standard of care for symptomatic lumbar disc herniations unresponsive to conservative care measures including physical therapy, nonsteroidal anti-inflammatory drugs, activity modification, and interventional spinal injection treatments. ${ }^{1-5}$ In comparison to open laminectomy surgery, microdiscectomy is associated with fewer postoperative complications. However, a literature review shows that complications following lumbar microdiscectomy are not uncommon and should be expected as in Table 1.

Minimally invasive surgical techniques using intermuscular plane or tubular retractors for mini-open incisional exposures ${ }^{14-16}$ are commonly used for decompression and reconstructive fusion procedures with less approach-related access trauma and blood loss, and reduced surgical pain. Postoperative recovery is further expedited with the use of spinal endoscopes that traverse a smaller surgical access corridor with their working cannula. ${ }^{17-21}$ The combination of these developments has facilitated a substantial increase in the types of procedures being conducted in an ambulatory surgery center (ASC), ${ }^{14,15}$ a trend that is welcomed by more patients because of convenience and less burdensome treatments, and, moreover, is motivated by recent coverage and bundled payment guidelines $^{22}$ of insurance providers and governmental institutions and review boards intended to help stem the rise of health care cost. This observation is corroborated not only by the recent substantial increase of current procedural terminology (CPT) codes for spinal surgeries, including fusions eligible for payment in ASCs, but also by the addition of new endoscopic decompression CPT codes for spinal surgeries. ${ }^{23}$ The latter is of particular significance as reimbursement to the ASC has been set at a level intended to be commensurate with the slightly higher case cost generated by the need for capital purchases and maintenance of spine endoscopic equipment (eg, video towers, light sources, irrigation pumps), and instruments (such as endoscopes, rongeurs, drills). In addition, there is a higher use of disposables, including specialized drapes, radiofrequency probes, and laser fibers.

The implication is that lumbar endoscopic decompression procedures can be performed in an ASC setting while obtaining improved clinical outcomes, fewer postoperative complications, shorter intervals for return to work, faster social reintegration, shorter postoperative narcotic independence, and an overall reduced utilization of painkillers $^{24,25}$-all at a low cost and low risk. However, the burden of proof is still out on this stipulation and the current literature is lacking conclusive evidence substantiating the "low-risk, low-cost" hypothesis. Measuring quality of care with analysis of clinical outcomes in correlation with clinical practice variations and quality of health care delivery has become paramount in the context of rising costs and constrained resources that have to be wisely applied to an increasing number of patients. ${ }^{26}$ Objective and reliable outcome data have become increasingly relevant when communicating with patients, referring doctors, hospitals, ASCs, and insurance providers, as well as governmental institutions and review boards, to assess quality and costs of health care. Comparative performance data on individual hospitals and physicians by procedure may also limit the costs of health care while improving quality. ${ }^{27}$

This study aims to investigate whether the outpatient lumbar transforaminal decompression procedure, when performed in an ASC for herniated disc and spinal stenosis, can adequately address sciatica-type leg and low back pain symptoms with lower postoperative complication rates and fewer postoperative acute-care measures compared to reported microdiscectomy rates by differentiating 
Table 1. Common complications and their incidence reported with microdiscectomy.

\begin{tabular}{lc}
\hline Complication(s) & Rate \\
\hline Dural tears & $3 \%-4 \%^{6-8}$ \\
Cerebrospinal fistula & $0.1 \%{ }^{7}$ \\
Wrong level surgery & $1.2 \%-3.3 \%{ }^{9}$ \\
Wound infections & $2 \%-3 \%^{7,9,10}$ \\
Spondylodiscitis & $<1 \%^{6,11}$ \\
Significant blood loss & $5 \%{ }^{9}$ \\
Nerve root damage ranging from sensory dysfunction & $0.3 \% 0^{6,7,9}$ \\
$\quad$ to loss of motor strength (foot drop) & $0.05 \% \%^{7,9}$ \\
Life-threatening retroperitoneal vascular lesion & $0.1 \%-0.2 \%{ }^{6,11}$ \\
Epidural hematoma with new neurological deficits & $2.2 \%{ }^{7,9,12}$ \\
Thromboembolic complication, even under chemical & \\
$\quad$ thromboprophylaxis & $2 \%{ }^{13}$ \\
Persistent leg pain after adequate decompression due & \\
$\quad$ to intraoperative nerve root manipulation causing & \\
$\quad$ neurapraxia for days to weeks & \\
\hline
\end{tabular}

complications from failure to cure and other expected procedure-inherent sequelae.

\section{MATERIALS AND METHODS}

In 2006, the Center for Advanced Spine Care of Southern Arizona established an outpatient spinal surgery program for the treatment of lumbar herniated disc and spinal stenosis. The results presented in this paper are based on a retrospective review of patients seen between the years 2006 and 2015. Patients were worked up diagnostically by reviewing prior referrals from consulting physicians, pain management treatment programs, and interventional care, and integrating the information to determine what additional diagnostic study or nonoperative treatment measure may have been appropriate prior to considering surgical treatment. Patients were seen for lumbar radiculopathy, with and without claudication, and low back pain.

\section{Patient Population}

All patients in this case series provided informed consent. This retrospective study included 1839 consecutive patients (1072 female and 767 male) seen in clinic who underwent percutaneous endoscopic foraminotomy and microdiscectomy at 2076 levels in an outpatient ambulatory surgery setting. All surgeries were performed by the author. The mean follow-up was 33 months, ranging from 24 to 85 months at the time this study was concluded. The inclusion criteria were (1) clinical signs of unilateral lumbar radiculopathy, dysesthesias, and decreased motor function; (2) imaging evidence of foraminal or lateral recess stenosis (criteria described below) demonstrated on preoperative magnetic resonance images (MRIs) and computed tomography (CT) scans; (3) unsuccessful nonoperative treatment including physical therapy and transforaminal epidural steroid injections for at least 12 weeks; and (4) an age of 30-85 years. Patients exhibiting pain syndromes involving more than one dermatome or those who had bilateral symptoms, or showed segmental instability on preoperative extension flexion radiographs, or had severe central stenosis (less than $100 \mathrm{~mm}^{2}$ ) were excluded from this study. ${ }^{28}$ Inclusion/exclusion criteria were used with the intent of minimizing the effect of other confounding factors. Patients' average age was $50.7 \pm 18.8$ years

\section{Preoperative Workup and Clinical Follow-Up}

Radiographs, MRI, and CT images were obtained preoperatively for all surgical patients. Typically, patients returned for clinical follow-up at 6 weeks postoperatively, and at 3, 6, 12, and 24 months, respectively. After the 2-year follow-up appointment, patients were seen on an annual or biannual basis. The long-term follow-up after 2 years was less reliable and available for only $81 \%$ of patients at 3 years, and $68 \%$ at 4 years, postoperatively. Results reported herein were, therefore, computed from data obtained at 2-year follow-up. Primary clinical outcome measures were reductions in the visual analog scale (VAS) for leg pain ranging from no pain (0) to worst pain (10) and the Oswestry Disability Index, both done by the patient and by the treating surgeon (K.U.L.) using the Macnab criteria. ${ }^{29}$ Briefly, follow-up results were classified as excellent if the patient had little pain and returned to desired activities with few limitations. Outcomes were classified as good if the patient reported occasional pain or dysesthesias performing daily activities with minor restrictions and did not need any pain medication. Patients were assigned to 1 of the 2 remaining categories if their pain improved somewhat, but they continued to need pain medication (fair), or if their function worsened or they needed additional surgery to address their symptoms (poor).

\section{Radiologic Classification of Foraminal Stenosis}

Lee's classification of foraminal and lateral recess stenosis was used to define the location of the offending pathology within the neuroforamen by dividing it from medial to lateral into entry (dura to pedicle; zone 1), middle (medial pedicle wall to 
center pedicle; zone 2), and exit zone (center pedicle to lateral border of the facet joint; zone 3). ${ }^{30}$ Foraminal and lateral recess stenosis were stratified according to the main offending pathology: extruded herniated disc, disc bulge, and disc bulge with concomitant bony stenosis. Disc herniations were further classified as upward, downward, migrated, or centered around this disc space using Lee's 4zone classification. ${ }^{31}$ The trajectory for placement of the access working cannula was chosen to provide best access to the location of migrated extruded disc fragments while considering patient-specific anatomical limitations, such as high-riding ilium, vertical collapse in the degenerative spine, or transitional anatomy. In the entry zone, Lee described hypertrophy of the superior articular facet as the predominant pathology. ${ }^{30}$ In the middle zone, it was often due to an osteophytic process underneath the pars interarticularis, and in the exit zone due to a subluxed and hypertrophic facet joint. ${ }^{30}$ These classification systems have been previously applied by the author. ${ }^{17}$ The height of the posterior intervertebral disc and lumbar foramina was evaluated according to Hasegawa et al, ${ }^{32}$ who described a lumbar neuroforaminal height of $15 \mathrm{~mm}$ or more as normal and reduced posterior intervertebral disc height of 3 to $4 \mathrm{~mm}$ as suggestive of spinal stenosis. Preoperative sagittal and axial MRI and CT images were used to assess the location and extent of foraminal stenosis. Only patients with stenotic lesions (whether due to bony stenosis, extruded disc herniation, or contained disc bulge) producing a neuroforaminal width of $3 \mathrm{~mm}$ or less on the sagittal MRI and CT cuts or lateral recess height of $3 \mathrm{~mm}$ or less on the axial MRI and CT cuts were treated surgically. The degree of lumbar intervertebral disc degeneration was also graded using the MRI classification system published by Pfirrmann et $\mathrm{al}^{33}$ with grade I designating the MRI appearance of the surgical disc as "...homogeneous with bright hyperintense white signal intensity and normal disc height...," and grade $\mathrm{V}$ as an inhomogeneous disc "...with hypointense black signal intensity. There is no difference between the nucleus and annulus. The disc space is collapsed...."33

\section{Surgical Techniques}

All surgical procedures employed the transforaminal endoscopic approach using the epiduroscopic technique, ${ }^{31}$ in which the working sheath is placed into the lower portion of the neuroforamen, thus retracting and avoiding the exiting nerve root. No part of the cannula tip or the endoscope is positioned in the disc space. The surgical technique used by the author (originally popularized by Hoogland et al and Schubert et al) ${ }^{34,35}$ employs a foraminoplasty in patients with or without lateral stenosis for the treatment of herniated disc.

Procedures were performed in prone position under local anesthesia and sedation in all patients. In rare instances, where access to the L5-S1 neuroforamen was difficult due to a high-riding ilium, patients were positioned in the lateral decubitus position in an attempt to facilitate access to the neuroforamen. Techniques to define the skin entry point and the surgical trajectory have been described in previous literature. ${ }^{36-39}$ Generally, entry points were laterally at 7 to $9 \mathrm{~cm}$ at the L3-4 level, 8 to $10 \mathrm{~cm}$ at the L4-5 level, and 10 to $12 \mathrm{~cm}$ at the $\mathrm{L} 5-$ S1 level. In general, steeper coronal plane approach angles were used for downward-migrated extruded disc fragments. Conversely, shallower coronal plane approach angles were used for upward-migrated extruded disc fragments.

The targeted neuroforamen was accessed as follows: First, an 18-G (150 mm in length) spinal needle was inserted into the safe zone of Kambin's triangle bordered by the traversing nerve root medially, the exiting nerve root laterally, and the lower adjacent pedicle distally. ${ }^{36,37}$ Ideally, the targeting needle was placed on the lateral view into the lower portion of the neuroforamen or into the disc. On the anterior-posterior view, the needle tip should be at the medial interpedicular line. A steel guide wire was then inserted, and the $18-\mathrm{G}$ spinal needle was removed. Dilators, motorized drills, and trephines of increasing diameters were used for the foraminal decompression procedures. Additional cannulated reamers and drills (maxmorespine by Hoogland Spine Products, Unterföhring, Germany) measuring 7 and $9 \mathrm{~mm}$ in diameter intended to be used over a guide wire without the protective working cannula were available but rarely used to further minimize risk of dysesthesia of the exiting nerve root and irritation of its dorsal root ganglion (DRG).

For the foraminoplasty, bone from the hypertrophied superior and inferior articular process was removed with different instruments including endoscopic chisels, drills, Kerrison rongeurs, and percutaneous trephines. The endoscopic drills and rongeurs were deployed inside the center working 
cannula of the endoscope to lessen the risk of dysesthesia and irritation of the exiting nerve root and its DRG. In other words, the entire decompression was performed under continuous direct videoendoscopic visualization and no part of the decompression procedure was performed percutaneously with only indirect fluoroscopic imaging.

The foraminoplasty was facilitated by changing the trajectory of the instruments to aim for the compressive pathology identified on preoperative studies. In other words, during foraminoplasty the bony decompression was focused on removing the relevant stenotic process identified on preoperative imaging studies using categories of the Lee classification (ie, in the exit, mid-, and entry zones). For example, a stenotic process in the entry zone would typically require a more substantial subtotal resection of the superior articular process starting at the tip of the process to be able to access the lateral recess. In case of concomitant herniated disc, extruded disc material was removed using forceps and pituitary rongeurs and contained herniations were decompressed through a small annular window. Epidural bleeding was controlled with a radiofrequency probe (Ellman International LLC, Baldwin, New York) under saline irrigation.

\section{Postoperative Rehabilitation and Utilization}

Postoperative rehabilitation and supportive care requirements were recorded and analyzed in relation to clinical outcomes with the secondary transforaminal endoscopic decompression procedure in lumbar mono-radiculopathy patients due to lateral stenosis, with or without herniated disc. During their regular postoperative visits, patients were asked whether they participated in any active exercise programs, physical or occupational therapy, chiropractic care, or had acupuncture or spinal injection treatments. In addition, the patient's utilization of nonsteroidal anti-inflammatories, narcotic, and other types of pain medication were recorded. Finally, patients were asked whether they developed any new pain syndromes, or hitherto unknown conditions that negatively impacted their walking endurance.

\section{Complication Analysis}

As conclusive assessments of clinical outcomes and cost of a surgical procedure hinge on consensus of how to define complications and to stratify them by severity, the recently modified classification system of Dindo et $\mathrm{al}^{26}$ was used in this study. Grading classification is as follows (complications refer to any deviation from the expected postoperative course):

Grade I: Complication implies that no pharmacological, surgical, endoscopic, or radiological interventions were necessary to manage the patient except "... antiemetics, antipyretics, analgesics, diuretics, electrolytes, and physiotherapy." ${ }^{26}$ Wound infections drained and treated at the bedside are also encompassed by a grade I complication. ${ }^{26}$

Grade II: Complications require additional pharmacological treatment as well as blood transfusions or total parenteral nutrition. ${ }^{26}$

Grade III: Complications require "... surgical, endoscopic, or radiological intervention." 26

Grade IIIa: Complications can be managed without general anesthesia.

Grade IIIb: Complications require general anesthesia.

Grade IV: Complications involve the central nervous system or require intensive care unit management, considered life-threatening. ${ }^{26}$

Grade IVa: Complications refer to organ dysfunction with a single organ failure (ie, requiring dialysis).

Grade IVb: Complications refer to a multiorgan failure.

Grade V: Complications leading to the patient's death. $^{26}$

In addition to the above complications, other types of negative postoperative outcomes including failure to cure, and expected postoperative sequelae were recorded in distinct categories. For this study, a complication was defined as any deviation from the normal postoperative course. ${ }^{26}$ Sequelae, on the other hand, were defined as possible expected "aftereffects" 26 from the transforaminal decompression surgery that were inherent to the procedure. According to the Dindo et $\mathrm{al}^{26}$ classification of surgical complications employed herein, failure to cure following a well-executed transforaminal endoscopic decompression surgery in patients suffering from symptomatic herniated disc or lateral recess stenosis without achieving pain relief did not constitute a complication, and, hence, was not included in the analysis of complications. Likewise, sequelae including extravasation of irrigation fluid into the spinal canal or in the subcutaneous tissues 
causing spinal headaches, or increased incisional pain, or pain from contusion of the ilium during the L5-S1 transforaminal approach, or dysesthetic leg pain from DRG irritation were also not included in the analysis of complications. Furthermore, a recurrent disc herniation, regardless of when it occurred in the postoperative course during the 2year study, was considered a grade I complication if it was managed without additional surgery, and a grade III complication if acute symptoms had to be managed surgically.

All complications during the stay at the ASC were recorded. As all patients enrolled in this study were discharged to return home after surgery, patients were asked at each follow-up visit whether they were treated for any postoperative complications in an emergency room (ER) and if any of these visits resulted in an admission to a hospital. Patients were also monitored during regular scheduled follow-up visits for any signs of unbeknownst or asymptomatic postoperative complications, such as arrhythmia or atelectasis. Complications during the first 6 weeks after the index surgery were of interest if they resulted in any visit to the ER or admission to a hospital. These visits were considered acutecare visits. Any ER visits or hospital admissions after 6 weeks from the transforaminal index decompression procedure were considered nonacute, chronic-care visits. The severity of the postoperative complications was graded and analyzed in relation to the patient's overall functional outcome.

\section{Unintended Care Cost Analysis}

The direct cost of unintended aftercare was estimated by obtaining 2018 cost data from the health network operating the hospital where patients were evaluated in the ER, admitted for observation for pain control or inpatient stay for additional workup with diagnostic studies, or had other nonsurgical and surgical treatments. Cost categories included ER visits, general radiology, global inpatient, observation inpatient, and inpatient neurology including surgical treatment. Payments received for these 5 categories were also recorded.

\section{Statistical Methods}

For the clinical outcomes analysis, cross-tabulation statistics and measures of association were computed for 2-way tables using IBM SPSS
Table 2. Patients by diagnosis $(n=1839)$.

\begin{tabular}{lr}
\hline Indication for Surgery & No. of Patients \\
\hline Lumbar disc herniations & \\
$\quad$ Extruded & 331 \\
Contained & 648 \\
Subtotal & 979 \\
Lateral recess stenosis & 860 \\
Total & 1839 \\
\hline
\end{tabular}

statistics software, Version 25 (IBM, Armonk, New York). Using patient satisfaction data and clinical outcomes data based on the modified Macnab criteria, VAS, type of complication, foraminal zone classification, MRI classification of disc degeneration, definition of the location of any herniated disc, and foraminal height and width parameters as row and column variables, and age (over 50 and under 50) as control variable (layer factor), the cross-tabulation procedure was employed to form one panel of associated statistics and measures for each value of the layer factor (or a combination of values for 2 or more control variables). This correlation matrix allowed calculation of variable combinations if no association was found between complications, clinical outcome and variable distribution was equal. Both the Pearson $\chi^{2}$ and the likelihood-ratio $\chi^{2}$ tests were used as statistical measures of association.

\section{RESULTS}

Of the 1839 patients that underwent outpatient decompression between 2006 and 2015 (Table 2), excellent and good results according to the Macnab criteria were obtained in $82.2 \%$ of patients with extruded disc fragments (331/1839). In this group, the mean VAS score decreased from $5.9 \pm 2.5$ preoperatively to $2.4 \pm 1.8$ at the final follow-up $(P<.01)$.

Patients with contained disc herniations (648/ 1839) had excellent and good results $69.7 \%$ of the time. In this group, the mean VAS score decreased from $7.2 \pm 1.6$ preoperatively to $3.1 \pm 1.5$ at final follow-up $(P<.01)$.

In the spinal stenosis group (860/1839), $75 \%$ of patients had excellent to good results. There were no major intraoperative, approach- or anesthesia-related complications.

Grade I complications (any deviation without additional interventions) were observed in $4(0.22 \%)$ of the 1839 total study patients (Table 3). Two patients had incidental durotomies $(0.11 \%)$ in the 
Table 3. Postoperative complications, sequelae, failure to cure, and readmission rates $(n=1839)$.

\begin{tabular}{|c|c|c|}
\hline $\begin{array}{l}\text { Deviation From } \\
\text { Normal Postop Course }\end{array}$ & $\begin{array}{l}\text { No. of } \\
\text { Patients }\end{array}$ & Rate \\
\hline Complication & Total: 26 & Overall: $1.42 \%$ \\
\hline \multicolumn{3}{|l|}{ Grade I } \\
\hline Durotomy & 2 & $0.11 \%$ \\
\hline Foot drop & 2 & $0.11 \%$ \\
\hline \multicolumn{3}{|l|}{ Grade II } \\
\hline COPD exacerbation & 11 & $0.6 \%$ \\
\hline Superficial wound infection & 1 & $0.05 \%$ \\
\hline Discitis & 1 & $0.05 \%$ \\
\hline \multicolumn{3}{|l|}{ Grade IIIb } \\
\hline $\begin{array}{l}\text { Reherniation after discectomy } \\
\text { for extruded disc fragment }\end{array}$ & 9 & $0.5 \%$ \\
\hline Grade IV & 0 & $0 \%$ \\
\hline Grade V & 0 & $0 \%$ \\
\hline Sequelae & 320 & Overall: $17.4 \%$ \\
\hline Extravasation of irrigation fluid & 69 & $3.75 \%$ \\
\hline Spinal headaches & 8 & $0.44 \%$ \\
\hline Ecchymosis & 14 & $0.76 \%$ \\
\hline Dorsal root ganglion irritation & 229 & $12.45 \%$ \\
\hline Failure to cure & 80 & Overall: $4.35 \%$ \\
\hline Contained disc herniation & 41 & $2.23 \%$ \\
\hline Central and lateral recess stenosis & 39 & $2.12 \%$ \\
\hline Acute-care readmissions (within 6 wk) & 16 & Overall: $0.87 \%$ \\
\hline Dorsal root ganglion irritation & 9 & $0.49 \%$ \\
\hline Infection & 2 & $0.11 \%$ \\
\hline Poor pain control & 5 & $0.27 \%$ \\
\hline Cumulative risk of adverse postop event & 442 & $24.04 \%$ \\
\hline
\end{tabular}

Abbreviations: COPD, chronic obstructive pulmonary disease.

traversing L5 nerve root sleeves (Figure 1). Both durotomy patients had epidural adhesions of herniated disc. One of these 2 patients had a rootlet herniation that was reduced intraoperatively. Both patients were successfully treated with an intraoperative blood patch through the endoscope and bed rest for 24 hours. Both patients had transitory decreased sensory function without motor deficit, and neither complained of spinal headaches.

Two patients immediately developed foot drop postoperatively on the surgical side $(0.11 \%)$ - both after a L4-5 decompression surgery for lateral recess stenosis. These 2 patients were reassured and sent home from the ASC with a prescription for an ankle-foot orthosis. One patient had non-insulindependent diabetes mellitus, and the other one did not. The patient with non-insulin-dependent diabetes mellitus improved somewhat from a $2 / 5$ motor strength examination for extensor hallucis longus, and tibialis anterior muscles immediately postoperatively, to a $4 / 5$ motor strength at 9 months followup visit. This patient was initially managed with an ankle-foot orthosis and physical therapy focusing on strengthening both the extensor hallucis longus and tibialis anterior muscles and on improving proprioception in the affected extremity. The other patient with extensor hallucis longus and tibialis

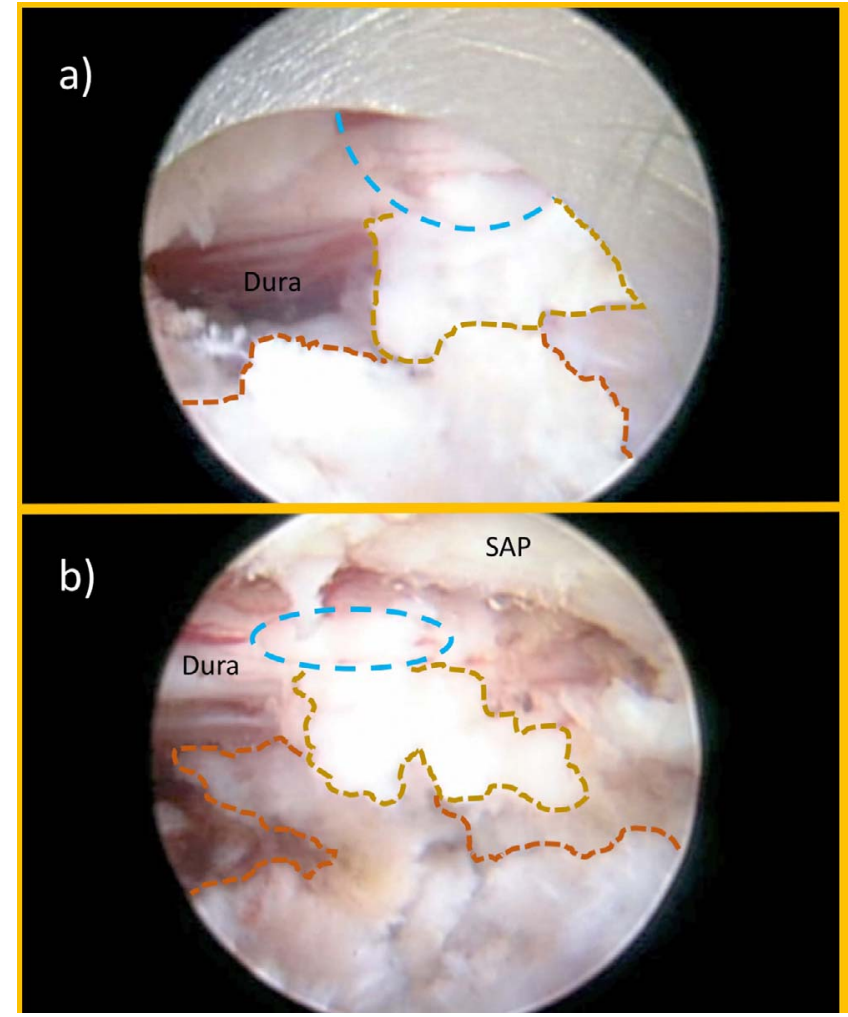

Figure 1. Intraoperative transforaminal endoscopic view of durotomy (blue dashed line) in the traversing $L 5$ nerve root sleeve. The remaining intervertebral disc (orange dashed line) is shown. A small adherent portion of the herniated disc (beige dashed line) below the traversing nerve root was common to both durotomy patients: (a) durotomy case I with oval durotomy, and (b) durotomy case II with a small longitudinal rent. In both cases, the rootlet herniations through the durotomy site were successfully managed with observation and bed rest. No attempt at repairing the durotomy was made in either patient. Abbreviation: SAP, superior articular process.

anterior weakness $(4 / 5)$ had transitory motor dysfunction that improved spontaneously within 6 weeks from the surgery with physical therapy and self-directed supportive care measures without needing an orthosis.

Grade II complications (any deviation with pharmacological interventions) occurred in 11 patients $(0.61 \%)$ with chronic obstructive pulmonary disease (COPD) who needed albuterol nebulizer treatment for decreased oxygen saturation in the recovery room (Table 3 ). Other comorbidities in these 11 medically complex patients included hypertension, coronary artery disease, type II diabetes mellitus, and obesity. Of the 11 COPD patients, 3 patients had to be transferred to a hospital to be medically stabilized but ultimately improved without intubation and were discharged from the ER after having been transferred directly from ASC to a hospital. Another 2 patients $(0.11 \%)$ had infections. These were successfully treated with oral antibiotics in one patient with a superficial 
wound infection, and with 6 weeks of intravenous antibiotics in another patient, who was diagnosed with a postoperative discitis.

Grade III complications (any deviation requiring surgical, endoscopic, or radiological intervention) were constituted by reherniations, which occurred in 9 of 331 patients (Table 3) with extruded disc herniations $(2.7 \%$ recurrence rate). Reherniations were associated with preserved disc height of $>6$ $\mathrm{mm}(P<.02)$. Each of these 9 patients underwent subsequent surgery with microdiscectomy at the same surgical level under general anesthesia. Reherniations, therefore, were classified as grade IIIb complications.

Grade IV (organ dysfunction) and grade V (death) complications did not occur in this study.

Failure of pain relief without significant improvement of walking endurance occurred in 39 of the 860 patients (Table 3 ) with bony stenosis in the central canal, lateral recess, and entry zone of the neuroforamen $(4.5 \%)$ and in 41 of the 648 patients with contained disc bulges $(6.3 \%)$. The latter subset of patients with contained disc herniation had advanced degenerative changes. According to the MRI grading system published by Pfirmann et $\mathrm{al}^{33}$ nearly all failure-to-cure patients with contained disc herniations had advanced degeneration of the surgical lumbar disc.

Sequelae occurred in 320 patients (Table 3). Sixty-nine patients had extravasations of irrigation fluid into the subcutaneous tissues $(3.8 \%)$. This was typically treated with a lumbar overlap corset and ice packs to the surgical area. The irrigation fluid within the subcutaneous tissues was reported by most patients to have dissipated within one or 2 days postoperatively. Another 8 patients developed spinal headaches $(0.4 \%)$ presumably from rostral spread of irrigation fluid into the spinal canal during surgery. Symptoms were managed with analgesics and antiemetics, when needed. All 8 patients were discharged from the ASC with instructions to bed rest for 24 hours. An additional 14 patients $(0.76 \%)$ had ecchymosis around the incisional area, presumably from bony bleeding after the foraminal decompression for facet hypertrophy. This was typically inconsequential and not associated with more pain, and no treatment was required. Dysesthetic leg pain due to DRG irritation occurred in 229 patients $(12.4 \%)$ and was unrelated to case frequency but was associated with severe foraminal stenosis $(P<.01)$. All 229 patients improved with supportive care measures.

The 3 COPD patients admitted from the ASC directly to an ER for medical stabilization constituted an acute ER use rate of $0.16 \%$ for decompensated medical problems. In addition, there were $26(1.41 \%)$ acute unintended postoperative ER visits by patients after discharge from the ASC to their home within the first 6 postoperative weeks. Hence, the overall acute ER use rate in this study was $1.58 \%$ (29 patients). Ten of the 26 patients evaluated in the ER within 6 weeks from the index procedure were sent home after reassurance and successful management of dysesthetic leg pain (Table 3). However, $16(0.87 \%)$ patients were acutely readmitted to a hospital over the 9-year study period $(0.87 \%)$ : 9 for dysesthetic leg pain, 2 for wound infections (1 superficial and 1 discitis), and 5 for poorly controlled incisional pain. Of the 16 admitted patients, 10 patients received a postoperative MRI scan, whereas another 3 had a postoperative $\mathrm{CT}$ scan as part of their postoperative workup. None of these advanced imaging studies prompted any change in management.

Chronic-care ER visits occurred in 9 patients. Of these 9 patients, 4 patients were seen in the ER for poor pain control of underlying low back pain syndrome within 6 weeks to 6 months from their index transforaminal decompression procedure. Of these 4 patients, 2 were admitted to the hospital for pain control. The remaining 5 patients were seen within the 6 weeks to 6 months postoperative care interval for other conditions unrelated to the spine.

The unintended care cost of the 3 COPD patients that were admitted from ASC directly to ER and were discharged from ER to home on the day of surgery after medical stabilization was estimated to be $\$ 525(\$ 175 \times 3)$. The direct cost of unintended acute aftercare following discharge from the ASC to home per patient was $\$ 175$ due to ER visits, $\$ 1,902$ per observation inpatient, \$299 due to general radiology (including lumbar MRI or CT), and $\$ 4160$ for inpatient neurology (including revision microdiscectomy; Table 4). Therefore, the subtotal expenditure due to unintended care by category was $\$ 5075$ for ER visits $(\$ 175 \times 29$ patients), $\$ 26628$ for the 14 observation inpatients $(\$ 1902 \times 14)$ of the 16 admitted patients who were hospitalized for control of dysesthetic leg (9 patients) or poorly controlled incisional pain (5 patients). The remaining 2 of the admitted patients were hospitalized for workup and 
Table 4. Postoperative unintended acute-care cost and payment.

\begin{tabular}{|c|c|c|c|}
\hline $\begin{array}{l}\text { Type of Unintended Acute Care and } \\
\text { Cost/Payment }\end{array}$ & Type and No. of Care Episodes & $\begin{array}{c}\text { Total Cost of } \\
\text { Unintended Hospital Care }\end{array}$ & $\begin{array}{l}\text { Total Hospital Payment } \\
\text { of Unintended Care }\end{array}$ \\
\hline $\begin{array}{l}\text { Direct transfer from ASC to hospital } \\
\text { ER }\left(\$ 175 / \text { level } 4^{\text {a }} \$ 618\right)\end{array}$ & $\begin{array}{l}3 \text { (COPD patients discharged from ER } \\
\text { to home after medical stabilization } \\
\text { without inpatient admission) }\end{array}$ & $\$ 525$ & $\$ 1854$ \\
\hline $\begin{array}{l}\text { ER visit after discharge from ASC to } \\
\text { home }\left(\$ 175 / \text { level } 3^{\mathrm{b}} \$ 281\right)\end{array}$ & $\begin{array}{l}26 \text { (16 admitted to inpatient, } 10 \\
\text { discharged home from ER) }\end{array}$ & $\$ 4550$ & $\$ 7306$ \\
\hline General radiology $(\$ 299 / \$ 169)$ & $13(10 \mathrm{MRI}$ and $3 \mathrm{CT})$ & $\$ 3887$ & $\$ 2197$ \\
\hline Global inpatient (\$6513/\$10 538) & 2 (wound infections) & $\$ 26628$ & $\$ 21076$ \\
\hline Observation inpatient $(\$ 1902 / \$ 2694)$ & $\begin{array}{l}14 \text { (9 patients for control of DRG } \\
\text { irritation dysesthetic leg pain, } 5 \\
\text { patients for incisional pain) }\end{array}$ & $\$ 26628$ & $\$ 37716$ \\
\hline Inpatient Neurology $(\$ 4160 / \$ 7519)$ & 9 (revision microdiscectomy) & $\$ 37440$ & $\$ 67671$ \\
\hline Total & 67 & $\$ 99658$ & $\$ 137820$ \\
\hline
\end{tabular}

Abbreviations: ASC, ambulatory surgery center; ER, emergency room; COPD, chronic obstructive pulmonary disease; MRI, magnetic resonance image; CT, computed tomography; DRG, dorsal root ganglion.

${ }^{a}$ CPT Code 99283: ER visit entailing an expanded problem-focused history, an expanded problem-focused examination, and medical decision making of moderate complexity. Counseling and/or coordination of care with other providers or agencies. The presenting problem(s) are of moderate severity. Source: Adapted from American Hospital Association and American Health Information Management Association (http://library.ahima.org).

${ }^{\mathrm{b}}$ CPT Code 99284: ER visit entailing a detailed history, a detailed examination, and medical decision making of moderate complexity. Counseling and/or coordination of care with other providers or agencies are provided consistent with the nature of the problem(s) and the patient's and/or family's needs. The presenting problem(s) are of high severity, and require urgent evaluation by the physician but do not pose an immediate significant threat to life or physiologic function. Source: Adapted from

American Hospital Association and American Health Information Management Association (http://library.ahima.org).

treatment of their wound infections and incurred expenditures of $\$ 13026(\$ 6513 \times 2)$ as estimated by the direct cost for global inpatient stay. The direct cost of general radiology was $\$ 3887(\$ 299 \times 13$ for 10 MRIs and 3 CTs). The direct cost of the revision surgery in the 9 patients that underwent revision microdiscectomy for recurrent herniated disc was $\$ 37440(\$ 4160 \times 9)$ as estimated by the expenditure of the inpatient neurology stay. Hence, there were a total of 67 unintended care encounters as a result of having performed 1839 transforaminal endoscopic procedures with a total expenditure of $\$ 99658$ at the hospital where patients presented for acute postoperative after care measures following their ASC outpatient endoscopic transforaminal decompression. The total amount of payments received by the hospital for these services was $\$ 137820$ (Table 4).

\section{DISCUSSION}

This study shows that individual complication rates with the ASC outpatient transforaminal lumbar decompression surgery for sciatica-type low back and leg pain with respect to reherniations, wound infections, durotomies, and nerve root injuries are approximately 1 magnitude lower than equivalent reported complication rates with microdiscectomy, while delivering comparable clinical outcomes and lower readmission rates to an ER or hospital. The latter has been reported to be as high as $4.1 \%$ to $5.8 \%$ for outpatient ambulatory open or mini-open microdiscectomy. ${ }^{1,2,40}$ The lower com- plication and readmission rates after transforaminal lumbar decompression may be explained by a more direct and anatomical approach to the compressive pathology with less exposure-related pain. Other contributing factors may be related to technological advances allowing for more complex bony spinal stenosis problems to be treated endoscopically. This less burdensome procedure is better accepted by patients, who report higher satisfaction with outpatient surgery, presumably because of lower blood loss, fewer complications, earlier narcotic independence from surgery, and earlier return to desired activities, and work. ${ }^{4,17,40}$ Hence, it is not surprising that endoscopic outpatient lumbar spinal surgery has gained popularity and is done in many centers around the world. ${ }^{40}$

The clinical outcomes observed in this study are certainly comparable to previously published outcome studies. ${ }^{2}$ Success rates ranging from $70 \%$ to $80 \%$ have been reported as more realistic when outcomes were analyzed in terms of the following:

- VAS leg pain relief $(80 \%)^{2}$

- VAS back pain relief $(77 \%)^{2}$

- reduction in Oswestry Low Back Disability Index $(78 \%)^{2}$

- overall satisfaction with surgery outcome $(76 \%)^{2}$

- return to normal daily activities $(65 \%){ }^{2}$ and

- return to work $(61 \%)^{2}$

Except for extruded disc herniations, essentially all patients were treated for lateral recess stenosis. Given that excellent and good outcomes, as measured with Macnab criteria, for the majority 
of patients following the transforaminal decompression procedure in an ASC setting were similar to those expected if the patients were inpatients, it can be concluded that these surgeries can be effectively performed in an ASC setting. ${ }^{35-37}$ As sciatica and neurogenic claudication refractory to conservative care was the main reason for surgical intervention, reduction of leg pain was analyzed using a VAS as one of the primary outcome measures. There was a significant improvement in the VAS and the clinical outcome at final follow-up suggesting that the outpatient transforaminal endoscopic decompression procedures is effective for most patients that had unrelenting sciatica and claudication symptoms prior to surgery. Our clinical outcomes as measured by modified Macnab criteria are comparable to success rates reported by patients undergoing laminectomy for spinal stenosis. ${ }^{28,41}$

This study shows that serious grades IV and V complications with the procedure are uncommon and, in fact, did not occur. Grade I complications (any deviation without additional interventions) occurred in only 4 patients - 2 of whom had intraoperative durotomies (incidence $0.1 \%$ ); the remaining 2 patients developed postoperative foot drop. The durotomy patients were successfully managed without additional surgery. Ultimately, the durotomies were inconsequential and did not lead to any acute-care admissions to an ER or hospital. The 2 patients with transitory foot drop improved with supportive care measures - one with complete and the other with incomplete recovery of motor function-suggesting that the motor deficit was due to neurapraxia. These 2 patients were sent home from the ASC with a prescription for an ankle-foot orthosis and ultimately did well with "hands-on" outpatient management, presumably due to the robust preoperative education process preparing the patient for the rare but possible complication and close clinical follow-up. Admission to an ER or hospital from the ASC was deemed unnecessary in both patients as the directly visualized endoscopic decompression procedure was done for lateral recess stenosis without nerve root injury. Residual compression due to retained or migrated disc fragments because of manipulation during the decompression surgery was not suspected and postoperative neurapraxia was the most likely explanation.

Grade II complications (any deviation with pharmacological interventions) were due to COPD
(11 patients) and postoperative infections (2 patients). These 13 patients were managed medically. Of the 11 COPD patients, 8 patients responded favorably to albuterol nebulizers in the recovery room and only 3 needed transfer to a hospital to be medically stabilized. None of the COPD patients were reintubated. The 2 patients with wound infections were successfully treated, one with oral antibiotics for a superficial wound infection, and the second with intravenous antibiotics for postoperative discitis. None of the patients with grade II complications required additional interventions or surgeries.

Grade IIIb complications (any deviation requiring surgical, endoscopic, or radiological intervention under general anesthesia) due to reherniations ( 9 of 331 patients with extruded disc herniations; $2.7 \%$ recurrence rate) were managed with revision microdiscectomy. Patients with persistent or recurrent symptoms did not require any acute- or chronic-care admissions to an ER or hospital, presumably because of persistence or recurrence of familiar pain. Our recurrence rates for extruded disc herniations $(2.7 \%)$ was low and commensurate with previous reports. ${ }^{1,2,18-20,34-40}$ Grade IV (organ dysfunction) and grade $\mathrm{V}$ (death) complications did not occur in this study.

Failure of pain relief without significant improvement of walking endurance in the 39 of the 860 patients with bony stenosis in the central canal, lateral recess, and entry zone of the neuroforamen $(4.5 \%)$ and in the 41 of the 648 patients with contained disc bulges $(6.3 \%)$ did not constitute a complication. In fact, failure to cure for an otherwise well-executed surgery is not unique to the transforaminal decompression procedure and occurs at relatively well-established rates for each surgical intervention. Failure to achieve pain relief was, at least in part, due to the advanced degenerative changes of the intervertebral disc and the facet joint complex in these patients as evidenced by their advanced Pfirrmann grades IV and V status.

Sequelae defined as expected "aftereffects" 26 inherent to the procedure occurred in patients with extravasations of irrigation fluid into the subcutaneous tissues, spinal headaches, and dysesthetic leg pain. A postoperative nerve root irritation occurred in 229 of the 1839 operated patients. Given the high incidence of $12.4 \%$ of postoperative nerve root irritations, patients are educated in our clinic 
preoperatively about this common postoperative problem. Analysis showed that it was unrelated to case frequency, but associated with severe foraminal stenosis $(P<.01)$ at a statistically relevant level. Our data show that patients exposed to the additional nerve root manipulation required during the foraminoplasty are prone to developing a postoperative DRG irritation. With this educational program in place, most patients with postoperative DRG irritation were successfully managed in an office setting with a combination of oral medication including nonsteroidal anti-inflammatories, gabapentin, or pregabalin; transforaminal epidural steroid injection; and activity modification to a light walking schedule and reduced physical activity program. Patients were advised that narcotic pain medication is not an effective treatment for dysesthetic leg pain due to postoperative DRG irritation. Poorly coping patients that present to an ER for postoperative pain control are likely to get noncontributory MRI or CT scans that in our patient series failed to change clinical management. However, these studies are often ordered by ER physicians or admitting hospitalists typically unfamiliar with the transforaminal decompression procedure prior to contacting the operating surgeon. It is not surprising that the common postoperative sequela of unrelenting dysesthetic leg pain due to DRG irritation was the most common reason for unintended acute-care visits to an ER and was also the most common reason for readmission to a hospital postoperatively. However, the readmission rate of $0.87 \%$ over the 9 -year study period observed was low compared to readmission rates reported with traditional microdiscectomy $(4.1 \%$ to $5.8 \%){ }^{2}$ The other common postoperative sequelae were extravasations of irrigation fluid into the subcutaneous tissues and spinal headaches from the index endoscopic decompression surgery, both of which did not lead to any unintended acute-care admissions. In an attempt to minimize symptoms related to irrigation fluid dissipation into the spinal canal or subcutaneous tissues, this author subsequently adopted a protocol of either limiting the peak pressure on the irrigation pump to less than $40 \mathrm{~mm} \mathrm{Hg}$, or using gravityassisted irrigation without a pump. Surgeons should be mindful of the length of surgery and the total amount of irrigation fluid used and expect irrigation fluid-related sequelae in patients who require complex decompression surgeries.
Besides comparable clinical outcomes with the endoscopic outpatient lumbar transforaminal endoscopic decompression surgery when compared to traditional inpatient microdiscectomy, this surgical technique bears the upside of additional cost savings that can be realized by performing the surgery in an outpatient surgery center rather than in a hospital setting, where the costs of admitting the patient are by far higher. Hospitalization may further increase cost by greater postoperative complication rates due to higher hospital-acquired wound, urinary tract, and pulmonary infection rates. Additional problems may arise in the hospital from medication errors and deviation from the postoperative pain management or rehabilitation protocol as the surgeon may not have complete control over custody of the patient throughout the hospitalization.

The pain medication utilization problem is of significance in the context of the narcotic abuse epidemic in the United States ${ }^{42-45}$ and has prompted increasing scrutiny by patients, insurance providers, and governmental institutions and review boards to employ a more stringent medical necessity and costbenefit assessment systems to both inpatient and outpatient spinal surgeries. ${ }^{46}$ Utilization of evidenced-based treatments is demanded by many to keep the rising cost of lumbar spinal surgery in check at a time where there is a growing demand for these types of procedures because of an aging babyboomer population. ${ }^{47,48}$

Recently, incentives to provide more cost-effective, high-value spine care have been put in place in the form of new CPT codes making a large number of spinal surgeries contractually feasible in an outpatient ASC. ${ }^{23}$ Bundled payments for care improvement models have been implemented in total joint arthroplasty to reduce costs by allotting a fixed payment for an episode of care to incentivize hospitals to implement core measures to decrease length of stay and the incidence of wound infections, improve integrated management of medical comorbidities, and reduce visits to the ER or readmission to the hospital, as well as reducing the number of patients sent to inpatient rehabilitation facilities for continued postoperative care. ${ }^{49,50}$ Implementation of similar strategies may be on the horizon for the increasing number of ASC spinal surgery procedures, which makes understanding factors contributing to postoperative complications and readmissions crucial in the overall design of a value-based outpatient spine care program. ${ }^{22}$ 
Although a precise cost-benefit analysis of the outpatient transforaminal decompression versus traditional microdiscectomy was beyond the scope of this study, it is obvious that performing low-cost/ low-risk spinal endoscopy in an ASC realizes immense cost savings when comparing lower complication rates and fewer postoperative ER visits and hospital readmissions than reported with other spinal surgeries. It has been estimated that inadequate management of care transitions related to avoidable complications and unnecessary hospital readmissions was responsible for $\$ 25$ to $\$ 45$ billion in wasteful spending in 2011 alone. ${ }^{51}$ Analyzing the 111892 patients who underwent spinal surgery of the database of the National Surgical Quality Improvement Project for years 2012 to 2014, Piper et al found a reoperation rate of $3.1 \%$, and an unplanned readmission (UR) rate of $5.2 \%{ }^{52} \mathrm{Su}$ et al studied risk factors associated for 30-day UR and major perioperative complications after spine fusion surgery in adults using the same database. $^{53}$ Their study included 18602 patients. They found a $5.2 \%$ overall major perioperative complication rate and a rate of $4.4 \%$ per 30 persondays for URs related to the index surgery. ${ }^{53}$ Among the risk factors were patient's advanced age, complex surgeries, and higher costs associated with American Society of Anesthesiologists class. A similar multi-institutional National Surgical Quality Improvement Project study was done by Kim et al on 7016 patients who received lumbar decompression procedures in 2011 showing an overall UR rate of $4.4 \%{ }^{54}$ Modhia et al estimated UR rates after spinal stenosis decompression surgery in a $5 \%$ randomly selected sample of Medicare beneficiaries identified in 2005-09 Medicare claims. ${ }^{55}$ They found an overall 1-year readmission rate of $9.7 \%$ in patients undergoing decompression fusion and $7.2 \%$ in patients who had only decompression $(P=.03)$. At 2 years postoperatively, readmission rates went up to $14.6 \%$ and $12.5 \%$, respectively. Advanced age and the presence of multiple comorbidities were found to be the main reason for high complication and readmission rates in Medicare beneficiaries versus younger patients. Kocher et al investigated the reasons for postoperative ER visits within the first 30 days of discharge using the 200507 Medicare data. ${ }^{56}$ Their study included 2382514 unique patients undergoing 2456021 procedures at 4536 unique hospitals. Patients who underwent coronary artery bypass grafting had the highest
ER use (22.4\%), compared to patients who had undergone back surgery (291 710 unique patients), who had the lowest (12.2\%). A total of 29603 of the 291710 unique Medicare patients with back surgery, who presented to an ER were admitted to a hospital either from the same ED $(47.1 \%)$, or from a different facility $(52.9 \%)$. The overall readmission rate in Medicare patients following back surgery was $10.1 \%$ within 30 days from discharge. ${ }^{56}$ The most common reasons for readmission after back surgery were noted to be cardiovascular or respiratory $(21.7 \%)$ followed by procedural site complications $(20.4 \%)$ and infection $(11.3 \%)$. Hence, the overall readmission rate for postoperative cardiovascular or respiratory complications after back surgery in Medicare patients was $2.2 \%{ }^{56}$

The direct cost of inpatient lumbar microdiscectomy was estimated on the basis of data derived from the randomized and observational cohorts of the original Spine Patient Outcomes Research Trial study $^{57}$ (across 1, 2, and 4 years posttreatment) at \$20 585 for inpatient, and \$11 616 for outpatient lumbar microdiscectomy. ${ }^{58}$ The same study reported incremental cost of $\$ 35583$ to $\$ 38159$ for first-year revision at a rate of $0.06 \%$ after surgical treatment and $\$ 33389$ to $\$ 40385$ for $0.03 \%$ revision rate in subsequent years after surgical treatment. ${ }^{58}$ The estimated cost of revision microdiscectomy ( $\$ 4160$ per inpatient neurology stay) in the 9 patients with recurrent herniated disc was substantially lower than the reported numbers. ${ }^{58}$ It is unclear whether that is due to regional variations in hospital acquired cost or, more likely, due to the use of different assumptions during the estimated direct cost data analysis. The direct cost generated by complicationor sequela-related unintended aftercare of $\$ 99658$ in total during the 9-year study period of 1839 patients in the 5 direct cost categories analyzed (Table 4) seems astonishingly low when compared to reported direct cost data reported from the Spine Patient Outcomes Research Trial ${ }^{58}$ The overall net societal impact of the 67 unintended acute aftercare encounters is better assessed by payments received by the hospital, which totaled $\$ 137820$.

Our data clearly showed much lower across-theboard complication and readmission rates without any increase during the 2-year follow-up, suggesting that the outpatient transforaminal endoscopic decompression procedure done in an ASC for sciaticatype back and leg pain is an attractive value proposition because cost savings associated with a 
lower complication rates are not only due to procedure-related factors but in particular due to the much lower incidence of complications from acute worsening of underlying chronic medical conditions. Only 11 of 1839 patients $(0.6 \%)$ had postoperative COPD exacerbations and only 3 of those 11 patients needed transfer from the ASC directly to a hospital on the day of surgery, from where they were sent home after being medically stabilized in the ER without inpatient admission. The direct cost of the ER evaluation of our 3 patients transferred from the ASC to a hospital for COPD exacerbation using the hospital-reported direct cost assumptions was minimal $(\approx \$ 525)$. The overall ER use rate in this study was approximately 8 times lower $(1.58 \%$ versus $12.2 \%)$ than previously published. ${ }^{56}$ The ER use rate due to postoperative decompensation of underlying medical problems was 14 times lower $(0.16 \%$ versus $2.2 \%$ ) than previously reported. ${ }^{56}$ The readmission rate with the outpatient transforaminal endoscopic decompression in our study was 12 times lower than previously reported $(0.87 \%$ versus $10.1 \%) .{ }^{56}$ The infection rate in our study was 11 times lower than previously reported. 56 Hence, the hypothetical direct cost savings from reduced unintended aftercare by having performed an outpatient transforaminal endoscopic decompression instead of an inpatient microdiscectomy from avoided readmission for decompensated cardiovascular and pulmonary problems (\$91 182) or treatments of infection (\$71 643) totaled $\$ 162825$. These numbers were calculated using the direct cost data from the admitting hospital under the assumption that all patients were Medicare beneficiaries and would have been managed as inpatients. The actual cost savings may have been higher since payments received by the hospital for acute aftercare were higher than direct cost for global inpatient, observation inpatient, and inpatient neurology (Table 4) and reported as an average.

Clearly, the lumbar transforaminal endoscopic decompression procedure is by far less burdensome to the patient and realizes direct cost savings not just by virtue of being carried out in an ASC, but also due to its much lower individual complication rates in areas where disproportionate expenditures can easily be accumulated once inpatient management of complications is required. In other words, the most common complications and sequelae of lumbar transforaminal endoscopic decompression were distinctly different from those reported with inpatient microdiscectomy ${ }^{6-13}$ and were managed in an outpatient office setting and, thus, never registered as additional claims since acute postoperative care measures were covered and included in the 90-day global payment period during the first 6 postoperative weeks.

To overcome the limitations of our retrospective study, a more accurate complication-related direct as well as indirect cost analysis of endoscopic transforaminal discectomy in an ASC versus inpatient microdiscetomy should be performed as a prospective randomized multicenter study to arrive at higher-grade clinical evidence to substantiate the findings of this study. One such randomized controlled trial is currently underway in the Netherlands to compare the effectiveness and cost-effectiveness of percutaneous transforaminal endoscopic discectomy versus open microdiscectomy for patients with a symptomatic lumbar disc herniation. ${ }^{59}$ However, results have not been published. While it may be more appropriate to measure and compare the cost-effectiveness of these 2 different procedures in additional quality-adjusted life-years gained to assess the overall societal benefit, the actual direct cost and payment data for complication- and sequelae-related aftercare obtained in this study still seems highly tangible information in negotiations with clinical care facilities and insurers for any spine surgeon who is attempting to establish an outpatient spinal decompression program in an ASC.

\section{CONCLUSION}

Outpatient transforaminal endoscopic decompression surgery should be considered a viable alternative to inpatient open lumbar surgery to provide patients with pain relief from sciatica-type back and leg pain. This conclusion is based not only on favorable clinical results comparable to open decompression surgery, but also on the merits of lower complication rates. Although $24.04 \%$ of patients had some deviation from a completely uneventful postoperative course, most of these deviations $(21.75 \%)$ were constituted either by inconsequential unavoidable procedure-inherent sequelae $(17.4 \%)$, or by failure to cure $(4.35 \%)$. The actual cumulative surgical complication rate $(1.42 \%)$ was still lower than individually reported complication rates for each common complication after microdiscectomy. The individual complication rates with the transforaminal endoscopic decom- 
pression procedure for durotomy, infection, foot drop, and reherniation were approximately 1 magnitude lower than reported in the literature for microdiscectomy. These lower complication rates ultimately translate into cost savings that in the context of value-based health care mandated by patients, insurance providers, governmental institutions, and review boards will afford the spinal surgeon a modern platform to better compete in the dynamically changing health care environment.

\section{REFERENCES}

1. Singhal A, Bernstein M. Outpatient lumbar microdiscectomy: a prospective study in 122 patients. Can J Neurol Sci. 2002;29(3):249-252.

2. Asch HL, Lewis PJ, Moreland DB, et al. Prospective multiple outcomes study of outpatient lumbar microdiscectomy: should 75 to $80 \%$ success rates be the norm? J Neurosurg. 2002;96(1 Suppl):34-44.

3. Zahrawi F. Microlumbar discectomy. Is it safe as an outpatient procedure? Spine (Phila Pa 1976). 1994;19(9):10701074.

4. Bookwalter JW III, Busch MD, Nicely D. Ambulatory surgery is safe and effective in radicular disc disease. Spine (Phila Pa 1976). 1994;19(5):526-530.

5. Cares HL, Steinberg RS, Robertson ET, Caldini P. Ambulatory microsurgery for ruptured lumbar discs: report of ten cases. Neurosurgery. 1988;22(3):523-526.

6. Hernandez-Perez PA, Prinzo-Yamurri H. Analysis of the lumbar discectomy complications [in Spanish]. Neurocirugia (Astur). 2005;16(5):419-426.

7. Ramirez LF, Thisted R. Complications and demographic characteristics of patients undergoing lumbar discectomy in community hospitals. Neurosurgery. 1989;25(2):226-230; discussion 230-231.

8. Tafazal SI, Sell PJ. Incidental durotomy in lumbar spine surgery: incidence and management. Eur Spine J. 2005;14(3):287-290.

9. Kraemer R, Wild A, Haak H, Herdmann J, Krauspe R, Kraemer J. Classification and management of early complications in open lumbar microdiscectomy. Eur Spine J. 2003;12(3):239-246.

10. Barker FG II. Efficacy of prophylactic antibiotic therapy in spinal surgery: a meta-analysis. Neurosurgery. 2002;51(2):391-400; discussion 400-401.

11. Lawton MT, Porter RW, Heiserman JE, Jacobowitz R, Sonntag VK, Dickman CA. Surgical management of spinal epidural hematoma: relationship between surgical timing and neurological outcome. J Neurosurg. 1995;83(1):1-7.

12. Schizas C, Neumayer F, Kosmopoulos V. Incidence and management of pulmonary embolism following spinal surgery occurring while under chemical thromboprophylaxis. Eur Spine J. 2008;17(7):970-974.

13. Shamji MF, Shcharinsky A. Use of neuropathic pain questionnaires in predicting persistent postoperative neuropathic pain following lumbar discectomy for radiculopathy. $J$ Neurosurg Spine. 2016;24(2):256-262.

14. Clark AJ, Safaee MM, Khan NR, Brown MT, Foley KT.
Tubular microdiscectomy: techniques, complication avoidance, and review of the literature. Neurosurg Focus. 2017;43(2):E7.

15. Overdevest GM, Peul WC, Brand R, et al; Leiden-The Hague Spine Intervention Prognostic Study Group. Tubular discectomy versus conventional microdiscectomy for the treatment of lumbar disc herniation: long-term results of a randomised controlled trial. J Neurol Neurosurg Psychiatry. 2017;88(12):1008-1016.

16. Soriano-Sánchez JA, Quillo-Olvera J, Soriano-Solis S, et al. Microscopy-assisted interspinous tubular approach for lumbar spinal stenosis. J Spine Surg. 2017;3(1):64-70.

17. Lewandrowski KU. "Outside-in" technique, clinical results, and indications with transforaminal lumbar endoscopic surgery: a retrospective study on 220 patients on applied radiographic classification of foraminal spinal stenosis. Int $J$ Spine Surg. 2014;1:8.

18. Yeung AT, Yeung CA. Minimally invasive techniques for the management of lumbar disc herniation. Orthop Clin North Am. 2007;38(3):363-372.

19. Tsou PM, Alan Yeung C Yeung AT. Posterolateral transforaminal selective endoscopic discectomy and thermal annuloplasty for chronic lumbar discogenic pain: a minimal access visualized intradiscal surgical procedure. Spine $J$. 2004;4(5):564-573.

20. Tsou PM, Yeung AT. Transforaminal endoscopic decompression for radiculopathy secondary to intracanal noncontained lumbar disc herniations: outcome and technique. Spine J. 2002;2(1):41-48.

21. Yeung AT, Yeung CA. Advances in endoscopic disc and spine surgery: foraminal approach. Surg Technol Int. 2003;11:255263.

22. Mok JM, Martinez M, Smith HE; Association for Collaborative Spine Research Investigators. Impact of a bundled payment system on resource utilization during spine surgery. Int J Spine Surg. 2016;10:19.

23. Hignite J. Understanding the impact of the CMS 2017 ASC payment rule on spine procedures. https://www.beckerss pine.com/spine/item/34156-understanding-the-impact-of-thecms-2017-asc-payment-rule-on-spine-procedures.html. Accessed January 10, 2019.

24. Debono B, Sabatier P, Garnault V, et al. Outpatient lumbar microdiscectomy in France: from an economic imperative to a clinical standard-an observational study of 201 cases. World Neurosurg. 2017;106:891-897.

25. Hersht M, Massicotte EM, Bernstein M. Patient satisfaction with outpatient lumbar microsurgical discectomy: a qualitative study. Can J Surg. 2007;50(6):445-459.

26. Dindo D, Demartines N, Clavien PA. Classification of surgical complications: a new proposal with evaluation in a cohort of 6336 Patients and Results of a Survey. Ann Surg. 2004;240(2):205-213.

27. Daley J, Henderson WG, Khuri SF. Risk-adjusted surgical outcomes. Annu Rev Med. 2001;52:275-287.

28. Sengupta DK, Herkowitz HN. Lumbar spinal stenosis. Treatment strategies and indications for surgery. Orthop Clin North Am. 2003;34(2):281-295.

29. Macnab I. Negative disc exploration. An analysis of the causes of nerve-root involvement in sixty-eight patients. $J$ Bone Joint Surg Am. 1971;53(5):891-903.

30. Lee CK, Rauschning W, Glenn W. Lateral lumbar spinal canal stenosis: classification, pathologic anatomy and surgical decompression. Spine (Phila Pa 1976). 1988;13(3):313-320. 
31. Lee S, Kim SK, Lee SH, et al. Percutaneous endoscopic lumbar discectomy for migrated disc herniation: classification of disc migration and surgical approaches. Eur Spine $J$. 2007;16(3):431-437.

32. Hasegawa T, An HS, Haughton VM, Nowicki BH. Lumbar foraminal stenosis: critical heights of the intervertebral discs and foramina. A cryomicrotome study in cadavera. $J$ Bone Joint Surg Am. 1995;77(1):32-38.

33. Pfirrmann CW, Metzdorf A, Zanetti M, Hodler J, Boos N. Magnetic resonance classification of lumbar intervertebral disc degeneration. Spine (Phila Pa 1976). 2001;26(17):1873-1878.

34. Hoogland T, Schubert M, Miklitz B, Ramirez A. Transforaminal posterolateral endoscopic discectomy with or without the combination of a low-dose chymopapain: a prospective randomized study in 280 consecutive cases. Spine (Phila Pa 1976). 2006;31(24):E890-E897.

35. Schubert M, Hoogland T. Endoscopic transforaminal nucleotomy with foraminoplasty for lumbar disk herniation. Oper Orthop Traumatol. 2005;17(6):641-661.

36. Kambin P, Casey K, O’Brien E, Zhou L. Transforaminal arthroscopic decompression of lateral recess stenosis. J Neurosurg. 1996;84(3):462-467.

37. Kambin P, O'Brien E, Zhou L, Schaffer JL. Arthroscopic microdiscectomy and selective fragmentectomy. Clin Orthop Relat Res. 1998;(347):150-167.

38. Kim MJ, Lee SH, Jung ES, et al. Targeted percutaneous transforaminal endoscopic diskectomy in 295 patients: comparison with results of microscopic diskectomy. Surg Neurol. 2007;68(6):623-631.

39. Ahn Y, Lee SH, Park WM, Lee HY, Shin SW, Kang HY. Percutaneous endoscopic lumbar discectomy for recurrent disc herniation: surgical technique, outcome, and prognostic factors of 43 consecutive cases. Spine (Phila Pa 1976). 2004;29(16):E326-E332.

40. Fallah A, Massicotte EM, Fehlings MG, et al. Admission and acute complication rate for outpatient lumbar microdiscectomy. Can J Neurol Sci. 2010;37(1):49-53.

41. Fokter SK, Yerby SA. Patient-based outcomes for the operative treatment of degenerative lumbar spinal stenosis. Eur Spine J. 2006;15(11):1661-1669.

42. Zolot J. A worsening opioid epidemic prompts action. Am J Nurs. 2017;117(10):15.

43. Cheatle MD. Facing the challenge of pain management and opioid misuse, abuse and opioid-related fatalities. Expert Rev Clin Pharmacol. 2016;9(6):751-754.

44. Hupp JR. The surgeon's roles in stemming the prescription opioid abuse epidemic. J Oral Maxillofac Surg. 2016;74(7):1291-1293.

45. Kee JR, Smith RG, Barnes CL. Recognizing and reducing the risk of opioid misuse in orthopaedic practice. $J$ Surg Orthop Adv. 2016;25(4):238-243.

46. O'Lynnger TM, Zuckerman SL, Morone PJ, Dewan MC, Vasquez-Castellanos RA, Cheng JS. Trends for spine surgery for the elderly: implications for access to healthcare in North America. Neurosurgery. 2015;77(Suppl 4):S136-S141.

47. Devin CJ, Chotai S, Parker SL, Tetreault L, Fehlings MG, McGirt MJ. A cost-utility analysis of lumbar decompression with and without fusion for degenerative spine disease in the elderly. Neurosurgery. 2015;77(Suppl 4):S116-S124.

48. Adogwa O, Parker SL, Shau DN, et al. Cost per qualityadjusted life year gained of revision neural decompression and instrumented fusion for same-level recurrent lumbar stenosis: defining the value of surgical intervention. J Neurosurg Spine. 2012;16(2):135-140.

49. Siddiqi A, White PB, Mistry JB, et al. Effect of bundled payments and health care reform as alternative payment models in total joint arthroplasty: a clinical review. J Arthroplasty. 2017;32(8):2590-2597.

50. Sullivan R, Jarvis LD, O’Gara T, Langfitt M, Emory C. Bundled payments in total joint arthroplasty and spine surgery. Curr Rev Musculoskelet Med. 2017; 10(2):218-223.

51. Colla CH, Wennberg DE, Meara E, et al. Spending differences associated with the Medicare Physician Group Practice Demonstration. JAMA. 2012;308(10):1015-1023.

52. Piper K, DeAndrea-Lazarus I, Algattas H, et al. Risk factors associated with readmission and reoperation in patients undergoing spine surgery. World Neurosurg. 2017;110:e627-e635.

53. Su AW, Habermann EB, Thomsen KM, Milbrandt TA, Nassr A, Larson AN. Risk factors for 30-day unplanned readmission and major perioperative complications after spine fusion surgery in adults: a review of the national surgical quality improvement program database. Spine (Phila Pa 1976). 2016;41(19):1523-1534.

54. Kim BD, Smith TR, Lim S, Cybulski GR, Kim JY. Predictors of unplanned readmission in patients undergoing lumbar decompression: multi-institutional analysis of 7016 patients. J Neurosurg Spine. 2014;20(6):606-616.

55. Modhia U, Takemoto S, Braid-Forbes MJ, Weber M, Berven SH. Readmission rates after decompression surgery in patients with lumbar spinal stenosis among Medicare beneficiaries. Spine (Phila Pa 1976). 2013;38(7):591-596.

56. Kocher KE, Nallamothu BK, Birkmeyer JD, Dimick JB. Emergency department visits after surgery are common for Medicare patients, suggesting opportunities to improve care. Health Aff (Millwood). 2013;32(9):1600-1607.

57. Weinstein JN, Lurie JD, Tosteson TD, et al. Surgical versus nonoperative treatment for lumbar disc herniation: fouryear results for the Spine Patient Outcomes Research Trial (SPORT). Spine (Phila Pa 1976). 2008;33(25):2789-2800.

58. Koenig L, Dall TM, Gu Q, Saavoss J, Schafer MF. How does accounting for worker productivity affect the measured cost-effectiveness of lumbar discectomy? Clin Orthop Relat Res. 2014;472:1069-1079.

59. Seiger A, Gadjradj PS, Harhangi BS, et al. PTED study: design of a non-inferiority, randomised controlled trial to compare the effectiveness and cost-effectiveness of percutaneous transforaminal endoscopic discectomy (PTED) versus open microdiscectomy for patients with a symptomatic lumbar disc herniation. BMJ Open. 2017;7:e018230.

Corresponding Author: Kai-Uwe Lewandrowski, MD, 4787 E. Camp Lowell Drive, Tucson, AZ 85712. Phone: (520) 204-1495; Email: business@tucsonspine.com.

Published 22 February 2019.

This manuscript is generously published free of charge by ISASS, the International Society for the Advancement of Spine Surgery. Copyright (C) 2019 ISASS. To see more or order reprints or permissions, see http://ijssurgery.com. 